\title{
PRÉSENCE EN SEINE-ET-MARNE \\ DE PHLEBOTOMUS LARROUSSEI
}

\author{
Par G. LAVIER et A. RISTORCELLI
}

Un exemplaire femelle de Phlebotomus larroussei Langeron et Nitzulescu 1931 a été capturé ie 2 août 1937 à Barbizon (Seineet-Marne) dans une habitation, à dix heures du soir, par l'un de nous (G. L.) qu'il se préparait à piquer.

\section{Description de L'exemplaire de Barbizon}

Taille. - $2.607 \mu$. L'exemplaire n'a pas été traité par la potasse, ce qui peut expliquer la brièveté de sa taille, Langeron et Nitzulescu avaient obtenu pour leurs exemplaires des tailles variant de 2.650 à $3.280 \mu$.

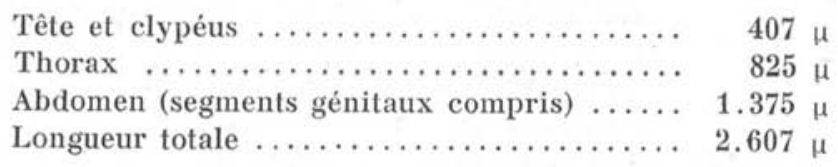

\section{Antennes :}

$$
\begin{aligned}
& \text { III }>\text { IV }+ \text { V } \\
& \text { III }<\text { IV }+ \text { V }+ \text { VI }
\end{aligned}
$$

Les épines géniculées sont bigéminées (pl. XXVII, fig. 2), leur extrémité ne dépasse pas le troisième quart de l'article, la formule antennaire est du type $\frac{2}{\text { III-XV }}$.

Palpes. - Formule palpale : 1, 4 (2, 3), 5. Rapport moyen des articles du premier au cinquième dans l'ordre : 1-3,9-4-3,3-7,7.

Longueur des articles :

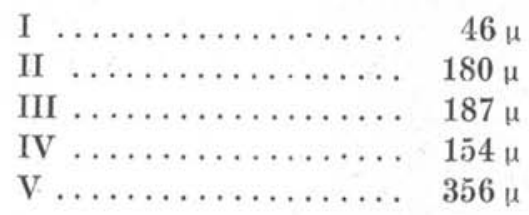

Annales de Parastrologie, T. XVII, N ${ }^{\circ}$ 5. - 1939-1940, p. 375-379. 
Les épines modifiées de Newstead sont situées à la partie moyenne du $3^{\mathrm{e}}$ article.

Epipharynx. - Longueur de $320 \mu$. Le rapport $\frac{\mathrm{A} \text { III }}{\mathrm{E}}=\frac{324}{320}=1$.

Cavité buccale. - Ni armature buccale, ni tache pigmentée.

Pharynx. - Il mesure $208 \mu$ de long, la largeur maxima, mesurée dans la partie distale, est de $61 \mu$. Il est donc environ trois fois plus long que large. L'armature pharyngienne ( $a$, fig. 1) occupe la partie postérieure de l'organe sur environ le quart de sa longueur

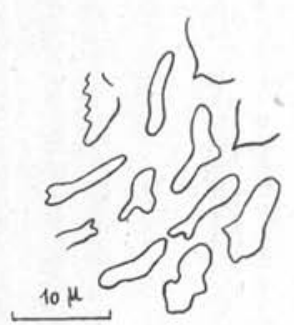

a

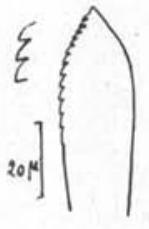

$b$
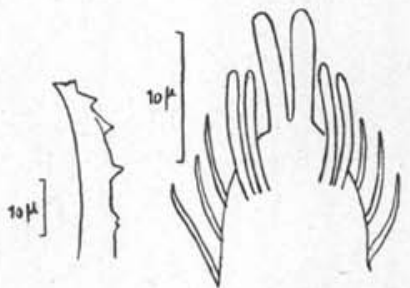

d

Fig. 1. - $a$, denticulation de l'armature pharyngienne ; $b$, mandibule ; $c$, maxille ; $d$, épipharynx.

totale, elle est constituée par un semis d'éléments saillants dont le contour diffère même pour chacun d'eux à l'immersion selon le déplacement de la vis micrométrique.

Pièces buccales. - Les pièces buccales (fig. 1) des phlébotomes ne sont pas décrites par tous les auteurs. Perfiliew avait cependant montré tout l'intérêt que présentait leur morphologie comparée (1928). Le labre-épipharynx se termine par deux grandes palettes; en retrait, et l'encadrant, deux autres paires de palettes aussi longues, mais beaucoup plus étroites. Le maxille rappelle par sa forme générale le maxille de $P$. chinensis étudié par Nitzulescu ; mais, à son extrémité apicale et sur le côté externe, en plus de quatre fortes dents chitinisées, il porte une cinquième dent, moins prononcée, paraissant constituée de deux petites denticulations jumelées. La mandibule ne semble pas spécifique de $P$. larroussei.

Aile. - L'aile (pl. XXVII, fig. 3) est relativement grande. Sa longueur est de $2 \mathrm{~mm} ., 05$; sa largeur de $0 \mathrm{~mm}$., 72, le rapport 


\section{$\frac{\text { taille de l'insecte }}{\text { longueur de l'aile }}=1,26$}

Elle est presque trois fois plus longue que large :

$$
\frac{\text { longueur de l'aile }}{\text { largeur de l'aile }}=2,84
$$

Le rapport alaire $\frac{\alpha}{\beta}=2,2$. La valeur de ò est de $187 \mu$.

Pattes. - Les pattes postérieures manquent. Le rapport $\frac{\text { tibia }}{\text { fémur }}$ (Raynal) est pour la première paire de 1,3; pour la seconde de 1,5.

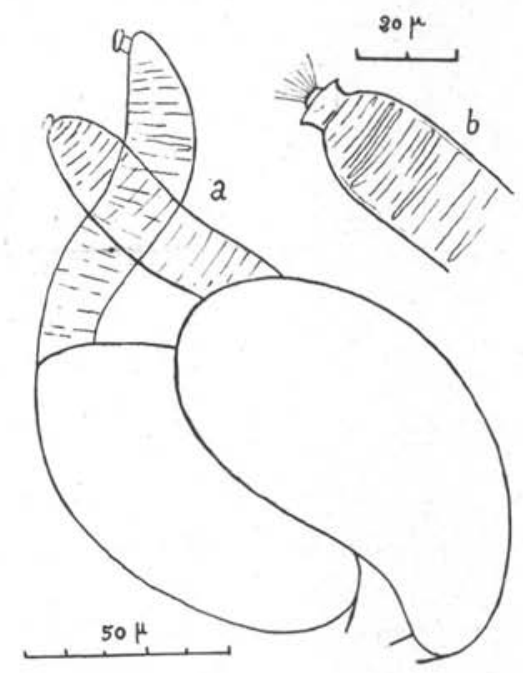

Fig. 2. - $a$, spermathèque du $P$. larroussei ; $b$, partie antérieure d'une spermathèque.

Langeron et Nitzulescu donnent comme rapports : 1,3 à 1,4 pour la première paire ; 1,5 à 1,6 pour la seconde paire ; 1,7 à 1,8 pour la troisième paire. Pour $P$. perniciosus, phlébotome le plus commun en France, les rapports sont de : 1,$1 ; 1,3 ; 1,5$.

Spermathèques. - Les spermathèques (fig. 2) sont de type un peu spécial, se rapprochant cependant du type strié. La tête est fixée à un cou court et émerge à peine de la collerette. L'ampoule, située dans la région postérieure de la spermathèque et caractéristique de l'espèce, ne paraît pas striée dans notre exemplaire. Les ampoules se terminent en conduits larges dont le commencement seul est 
visible. La partie antérieure de la spermathèque présente une ébauche de crénulations; les incisures qui sont à peu près parallèles sont peu profondes et discontinues. La largeur de la partie antérieure striée de la spermathèque est de $20 \mu$; la largeur de la partie ampullaire lisse est de $56 \mu$.

\section{RÉcapitulation des LOCalités de P. Iarroussei}

Cette espèce, signalée encore en France seulement, a été décrite par Langeron et Nitzulescu en 1931, d'après six exemplaires femelles dont quatre récoltés par Langeron depuis 1914 à Bourgla-Reine (Seine); le cinquième à Savignies près Beauvais (Oise) en 1921 ; le sixième à Varennes (Côte-d’Or) par Lesne en 1912.

Raynal et Le Gac ont capturé deux exemplaires femelles : l'un dans les Bouches-du-Rhône en 1932, à La Rose, dans la banlieue de Marseille; l'autre dans les Hautes-Pyrénées, en août 1933, à Capvern.

Enfin, un exemplaire femelle a été identifiẻ à Excideuil-sur-Vienne (Charente), en juillet 1935, par Colas-Belcour et Tisseuil.

\section{Sur LA Synonymie DE P. larroussei ET P. vesuvianus}

Adler et Theodor concluent à la synonymie des deux espèces. Cette manière de voir n'est pas acceptée par Langeron à cause de la différence de morphologie des spermathèques: spermathèques de $P$. vesuvianus sans dilatation ampullaire; spermathèques de $P$. larroussei avec dilatation ampullaire. La description de deux mâles provenant tous deux de l'île de Crète, l'un de Cato-Chorio (Parrot, 1937), l'autre de la Canée (Adler, Theodor et Witenberg, 1938), si elle complète définitivement les connaissances morphologiques de l'espèce $P$. vesuvianus, ne résout pas le problème de la synonymie des deux espèces. Il ne pourra l'être, comme l'écrit Parrot, qu'après identification du mâle encore inconnu en France

\section{EXPLICATION DE LA PLANCHE XXVII}

Phlebotomus larroussei.

FIG. 1. - Pharynx. Préparation originale.

FIG. 2. - Epines géniculées de l'antenne. Préparation originale.

Fıg. 3. - Aile. Préparation originale. 
ANNALES DE PARASITOLOGIE

T. XVII, No 5, 1939-1940

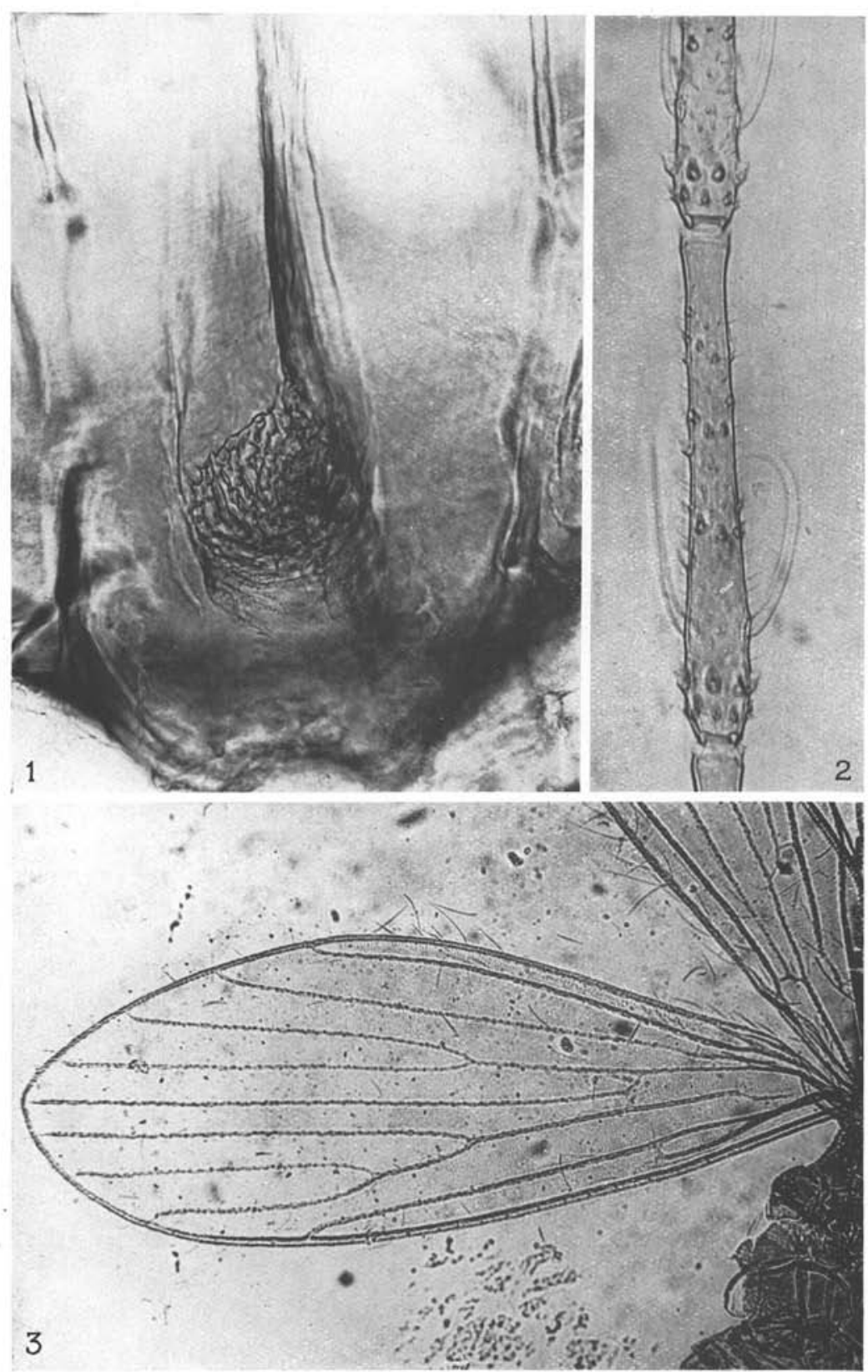

Masson et Cie, Editeurs
Planche XXVII

(Mémoire Laviek et Ristorcelli) 
de $P$. larroussei. On peut cependant penser dès maintenant qu'il s'agit de deux formes très voisines, le vesuvianus constituant très probablement une variété de la première espèce.

\section{BIBLIOGRAPHIE}

Anler (S.) et ThEodor (O.). - A study of the sandfly population in endemic foci of infantile kala-azar in Italy. Bull. entomol. research, XXII, 1931, p. 105 .

Adlen (S.), ThÉodor (O.) et Wrtenberg (G.). - Investigations on mediterranean kala-azar, XI. A study on leishmaniasis in Canea (Crete). Proc. Roy. Soc., B, CXXV, 1938. p. 491.

Colas-Bercour (J.) et Tisseuil (J.). - Nouvelle contribution à la répartition des phlébotomes en France. Rev. Path. comparée et Hyg. générale, 1936. p. 117.

Langeron (M.). - A propos du Phlebotomus larroussei. Ann. Parasit., X, 1932, p. 96.

Langeron (M.) et Nitzulescu (V.). - Phlebotomus larroussei n. sp., nouvelle espèce européenne de phlébotome. Ann. Parasit., IX, 1931, p. 72.

- Révision des phlébotomes de France. Ann. Parasit., X, 1932, p. 286.

Panrot (L.). - Notes sur les phlébotomes, Xix. Phlébotomes de Crète. Arch. Inst. Pasteur d'Algérie, XIV, 1936, p. 50.

- Notes sur les phlébotomes, XXiv. Sur le mâle de Phlebotomus vesuvianus Adler et Theodor 1931. Arch. Inst. Pasteur d'Algérie, XV, 1937, p. 104. Perfiliew (P.). - Zur vergleichende Anatomie von Phlebotomus. Zeitschrift für Parasitenkunde, I, 1928, p. 437.

Raynat (J.) et Le Gac (P.). - Sur un exemplaire de Phlebotomus larroussei Langeron et Nitzulescu 1931, capturé à La Rose (banlieue de Marseille). Ann. Parasit., X, 1932, p. 504.

Présence de Phlebotomus larroussei à Capvern (Hautes-Pyrénées). Ann. Parasit., XII, 1934, p. 343.

Institut de Parasitologie de la Faculté de médecine de Paris (Directeur: Prof. E. Brumpt). 